PIOTR KOMOROWSKI

Cardinal Stefan Wyszyński

University in Warsaw, Poland

p.komorowski@uksw.edu.pl

ORCID iD: 0000-0002-4731-271X

JACK WARCHALOWSKI

CMS Montera, Toronto, Canada

jack.warchalowski@cmsmontera.com
DOI: $10.13166 / j m s / 144166$

JOURNAL OF MODERN

SCIENCE TOM 2/47/2021

Piotr ZaWAda

Cardinal Stefan Wyszyński

University in Warsaw, Poland

p.zawada@uksw.edu.pl

ORCID iD: 0000-0003-2817-9578

\title{
THE ORGANIZATIONAL CULTURE OF ENTERPRISES AND CHANGES IN SUPPLY CHAIN MANAGEMENT IN THE COVID-19 PANDEMIC ERA
}

\begin{abstract}
The current economic situation brings many challenges for the functioning of enterprises. The global polarization of the world economy is progressing within the production and consumption poles as well as the centre of financial surpluses and the centre of debt. An additional factor having a significant impact on market realities is the ongoing COVID-19 pandemic. The high dynamics of economic processes forces enterprises to change management methods, operating strategies, the range of tools used, as well as changes necessary in organizational culture, not to mention application of new technologies. It can be said that we operate in the era of changes, so change management is becoming a dynamically developing area. The current economic challenges have significantly influenced the development of modern methods of supply chain management. The aim of the paper is to identify the changes in the approach to supply chain management in the pandemic era. The discussion is based on the analysis of source wmaterials, including industry reports, as well as the presentation of modern methods of supply chain management based on author's practical experience.
\end{abstract}

KEYWORDS: organisation culture, change management, supply chain management, COVID-19 pandemic, digital supply networks, push-pull strategy 


\section{THE PANDEMIC-DRIVEN EFFECTS ON ENTERPRISES IN SUPPLY CHAIN MANAGEMENT}

Many manufacturing and distribution organizations, especially now, face a significant challenge of managing their supply chains to ensure material supply. This challenge, recently enhanced by the global material shortage, normally narrows down to minimizing transportation / logistics costs ( Silva, Goncalves, Leite, 2014, pp. 329-343) while ensuring the right inventory levels at all strategic locations. Today's hyper-competitive environment(Biedenbach, Soderholm, 2008, pp. 123-145), characterized by constant change, market unpredictability, and the pressure to reduce costs and cycle times, coupled with the globalization trend, has provided further impetus to the growth of outsourcing (Kroes, 2007, p. 1) and development of the supply chain.

The need for more efficient supply chain management became even more apparent during the period of the COVID-19 pandemic. During this period, it was possible to observe that as the supply shocks intensified, global ports became increasingly congested. The delays in logistics led to further problems in regards to deliveries, production sustainability and even in retail trade all across the global economy.

Chart 1.

Containers waiting off Hong Kong-Shenzhen, based on vessels reporting status as anchored (2021).

Container Ships In Port / Container Ships Waiting

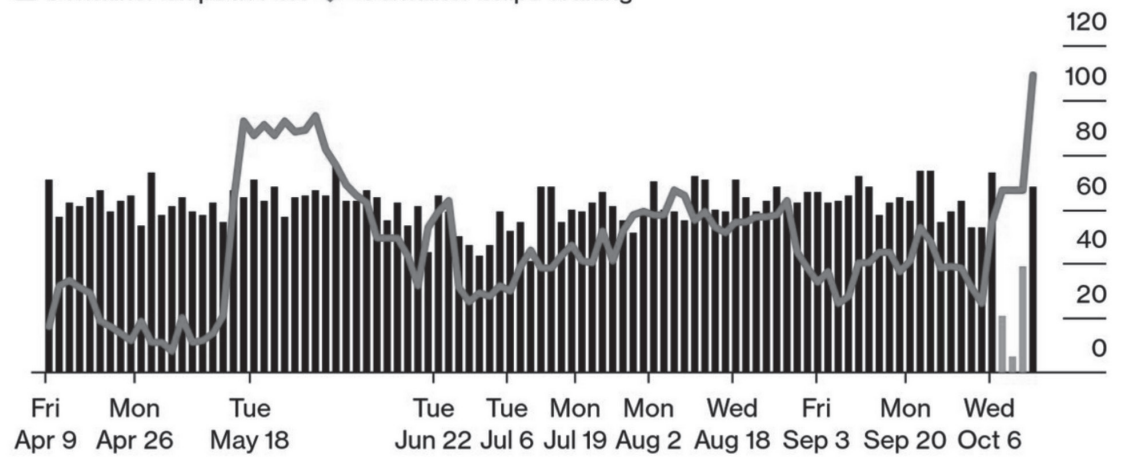

Source: Bloomberg, HIS Markit, Grenscape, from www.bloomberg.com (Access 25.10.2021) 
Ernst \& Young LLP led a survey of 200 senior-level supply chain executives in late 2020 in which topics including COVID-19 impact on supply chains and the development paths in supply management for the coming years. Out of the responding companies only $2 \%$ stated that were fully prepared for the pandemic. $72 \%$ of the responders reported a negative effect of the pandemic on their company, of which $17 \%$ highlighted that the effects were severe. The research indicated that immense changes are to be expected in regards to managing supply chains. Key, major factors of change in supply management for the following 3 years were indicated to be increase in efficiency (65\% of responders), reskilling supply chain models (61\%) and increase in supply chain visibility (61\%). Other factors were increase in responsiveness/resiliency, cost reduction, increase of sustainability and corporate social responsibility, larger diversity in supplies, move towards contract manufacturing and further application of digital technologies. $65 \%$ of the responders highlighted that digital transformation will accelerate due to the pandemic, including digital enablement and automation (52\% of responders mentioned the coming increase of the role of autonomous supply chain i.e.. robotised warehouses, driverless forklifts and trucks, development of drones and fully automated planning). According to the research, the supply chain of the future will need to be agile, flexible, efficient, resilient and digitally networked for improved visibility (Global board risk survey, 2020). As a result five priorities for recovery of organizations have been formulated (Global board risk survey, 2020):

1. Reimagine the strategic architecture of your supply chain:

- Rapidly redefine the supply chain strategy and alter global trade flows, considering new trade agreements, country incentives and omnichannel acceleration.

- $\quad$ Reimagine the supply chain operating model — what work should get done locally, regionally and globally, including warehouses and manufacturing sites. There are considerable tax implications here, and a new model can also help you prepare for future disruption.

2. Build transparency and resiliency:

- Improve disruption response with real-time visibility and monitoring of your end-to-end supply chain, as well as performing scenario planning and simulations. 
- $\quad$ Review the supply chain footprint with analysis of alternate sources of supply established and vendor or geographic concentration.

3. Extract cash and cost from your supply chain:

- Drive a step change in the supply chain cost structure and working capital profile by focusing on SKU rationalization, procurement spend reduction, logistics and warehouse optimization, and manufacturing productivity.

- $\quad$ Reduce working capital via supply chain segmentation, refreshed inventory planning parameters and changes in payment terms.

4. Create a competitive advantage with sustainability:

- The future is a circular economy (Korhopnen, Honkasalo, Seppälä, 2018, pp. 37-46, and Murray, Skene, Haynes, 2017, pp. 369-380, and Sachs, 2015) where there is no waste in your products or manufacturing.

- Explore ways to redesign and engineer new products to achieve this circular economy and monitor third-party risk with supplier sustainability assessments across tiers 1-3.

5. Drive agility and opportunities for growth through a digital supply chain:

- Work towards implementing the digital and end-to-end supply chain across planning, procurement, manufacturing and logistics. This can drive efficiencies and also open new revenue streams.

- Realize that companies are using supply chains as an engine for growth and a key differentiator versus competitors.

In another research made by Deloitte, it was highlighted that as China has become the "world factory", companies that are reliant on Tier 1 (direct) and Tier 2 (secondary) suppliers in China may experience significant disruptions in supplies during the pandemic (Covid-19, Managing supply chain risk and disruption. 2020). In the mentioned report it has been highlighted that due to the focus, which lasted decades, on minimization of costs, reduction in inventories and focus on drive up asset utilization, the safety buffers and flexibility and flexibility to absorb disruptions have been wiped out. The report highlights some key measures that companies could make to protect their supply chain, as per Table 1 . 
Table 1.

\section{Key measures for companies to protect their supply chain in the pandemic of} COVID-19

\begin{tabular}{|c|c|}
\hline $\begin{array}{l}\text { For companies that } \\
\text { operate or have } \\
\text { business relationships } \\
\text { in China and other } \\
\text { impacted countries }\end{array}$ & $\begin{array}{ll}\text { - } & \text { Educate employees on COVID-19 symptoms and prevention } \\
\text { - } & \text { Reinforce screening protocols } \\
\text { - } & \text { Prepare for increased absenteeism } \\
\text { - } & \text { Restrict non-essential travel and promote flexible working } \\
\text { - } & \text { Align IT systems and support to evolving work requirements } \\
\text { - } & \text { Prepare succession plans for key executive positions } \\
\text { - } & \text { Focus on cash flow }\end{array}$ \\
\hline $\begin{array}{l}\text { For companies that } \\
\text { produce, distribute, or } \\
\text { source from suppliers } \\
\text { in China and other } \\
\text { impacted countries }\end{array}$ & $\begin{array}{ll}\text { - } & \text { Enhance focus on workforce/labor planning } \\
\text { - } & \text { Focus on Tier } 1 \text { supplier risk } \\
\text { - } & \text { Illuminate the extended supply network } \\
\text { - } & \text { Understand and activate alternate sources of supply } \\
\text { - } & \text { Update inventory policy and planning parameters } \\
\text { - } & \text { Enhance inbound materials visibility } \\
\text { - } & \text { Prepare for plant closures } \\
\text { - } & \text { Focus on production scheduling agility } \\
\text { - } & \text { Evaluate alternative outbound logistics options and secure } \\
\text { - } & \text { Capacity }\end{array}$ \\
\hline $\begin{array}{l}\text { For companies that } \\
\text { sell products or } \\
\text { commodities to China } \\
\text { and other impacted } \\
\text { countries }\end{array}$ & 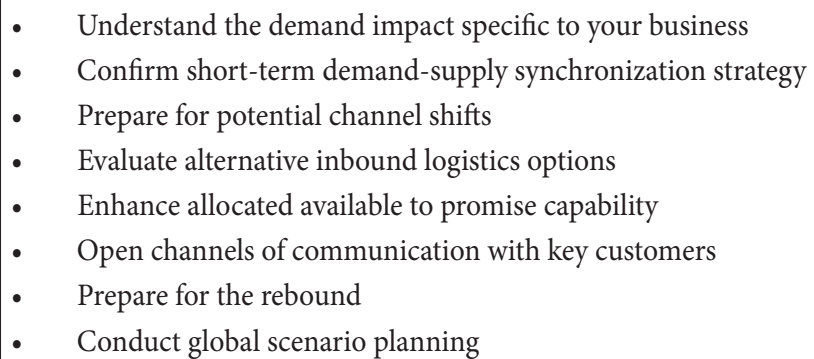 \\
\hline
\end{tabular}

Source: Covid-19, Managing supply chain risk and disruption, Deloitte Canada. 
In the dynamically changing economic environment, corporations turned out to be lumbering giants with rigid and over bloated management structures that in the end lacked the agility to adapt to the new conditions (Kalinzi, 2016, p. 3). The solution to the new, post pandemic economy in regards to the supply chain management may be in the new technologies such as digital supply networks (DSNs), where functional silos are broken down and organizations become connected to their complete supply network to enable end-to-end visibility, collaboration, agility, and optimization, without the traditional "overhead" associated with risk management techniques.

Chart 2.

\section{Traditional linear supply chain vs digital supply networks (DSNs)}

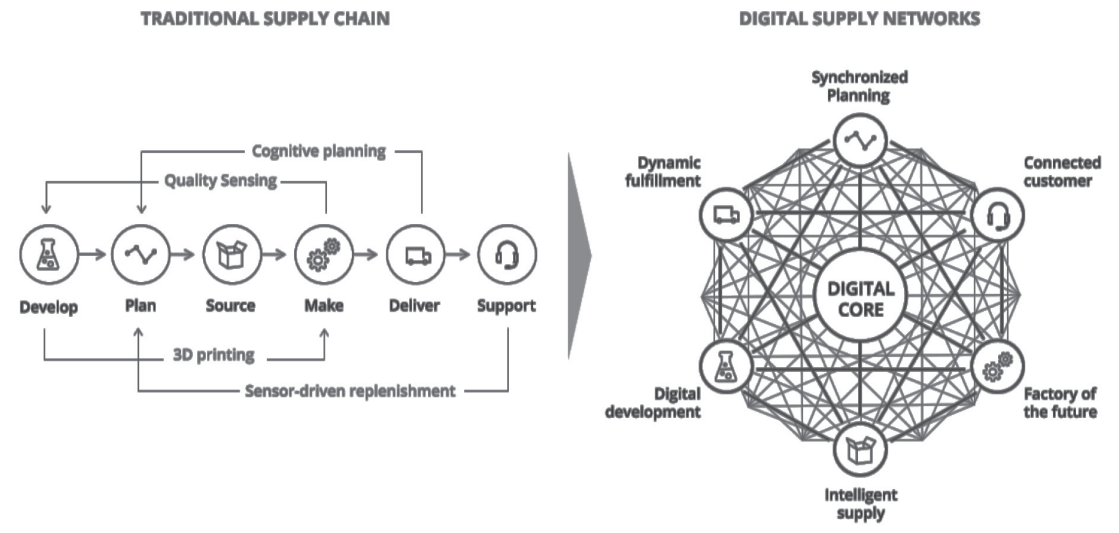

Source: Covid-19, Managing supply chain risk and disruption, Deloitte Canada.

The current wave of digital transformation is changing the business rules across the world in most production and services sector (Komorowski, 2020, pp. 329-343). The role of digital tools, including big data will be a crucial factor for the employees to run automated businesses. This development will also increase the dynamics in the changes in organization culture of the involved enterprises. 


\section{Push AND PUll STRATEgy}

All organizations that decide to manage inventory, meaning that their customers' tolerance time is shorter than their production or delivery lead time, utilize at least one of two primary supply chain strategies - push or pull (Minculete, Olar, 2016, pp.165-172). The most common one, the Push, relies on creating the best possible forecast of customer demand. This forecast gets translated down to the Stock Keeping Unit (SKU) level through the product Bill of Materials (BOM), specifying quantity for each raw material or component necessary for its production. Once generated, the buyers and planners can order needed supply and create forward looking master production plan necessary for the organization to function. This methodology, enabled by and relied on all MRP / ERP IT systems, has one fundamental flaw. Creating a reliable and accurate customer forecast became not only challenging but basically impossible as customer behaviour changed to more diverse and highly customized. The challenge isn't to predict how much overall sales a given business can generate and deliver. It is to describe precisely what specific products, in what quantities and when in terms of the future timing will be required. The more detailed the forecast level (closer to a given SKU) and the longer the timing, the bigger the forecast error. Buyer and planners, however, need this level of forecast to be able take daily actions and complete their jobs. Since this level of precision is impossible to achieve Push strategy normally results in higher than expected inventory levels, frequent material shortages, unsatisfactory customer service and higher expediated freight cost. In addition, many manufacturing plans suffer from increased firefighting, overtime, lower equipment utilization, all negatively impacting business productivity. Significant IT system training (for any ERP) and difficult to acquire worker's intuition, to correct forecast driven errors and its implications, is required to sustain this type of business operation.

The Pull strategy was developed to deal with the issues mentioned above. Instead of trying to create a perfect forecast, the Pull methodology defines a target inventory at a SKU level for a given location and then generates replenishment signal based on actual consumption (Kanban). If there is no consumption, there is no buying / producing. If there is more "pull" of a given 
item there is more frequent replenishment. This methodology created first by Ford Manufacturing and then mastered by the Toyota Production System managed to stabilize auto manufacturing production on all continents and became the start of Lean Manufacturing or Just in Time operation. From that point on it was used in many other industrial sectors from aerospace to electronics and even pharmaceuticals. This approach often relies on Visual Factory concepts, driven by Lean Thinking, and requires a significant training level of the manufacturing and supply chain teams. It works best with a limited diversity / complexity of finished products or their bill of materials. Linear assembly operations, like a car assembly, are the most suited to benefit from this approach. That's probably the main reason why the Pull approach was created first within the automotive supply chain.

\section{Plant Operations - VATI Analysis}

Another tool to be used to identify potential bottlenecks within a facility is the VATI Analysis. The VATI method allows to determine the best way to schedule and manage the enterprise's operations. Manufacturing plant operations can be grouped into four main types - V, A, T, I. While the main objective of this paper isn't to provide a detailed analysis of each plant type, a brief description of each and their fit with the supply chain strategies discussed above will be provided.

V Plants can be simply described as manufacturing operations where the number of finished products is much greater than the number of raw materials needed. A typical example of the V Plant can be an apparel manufacturer. From one type of fabric material, at one production step, you can cut a number of $\mathrm{t}$-shirt sizes that can later be further customized in terms of product colour, finishes, and other attributes. In its manufacturing routing sequence, there is usually several divergent points that create a V-like product flow. Apparel manufacturers typically have multiple finished SKUs (many T-shirt sizes, colours, etc.) and a smaller number of corresponding raw materials needed for their production. They can have, however, a huge number of other components (buttons, embroidery, etc.) needed to finalize the SKU. 
$\mathrm{V}$ plants are one of the most challenging environments to utilize a described above Pull replenishment approach. There are simply too many locations at all divergent points within the plant flow to establish appropriate Kanban locations and create a stable replenishment signal. The Push strategy is even more challenging due to the number of SKU level forecasts needed to make it reliable.

The next type of plants are the A Plants. When the number of components is much larger than the number of finished SKUs we are dealing with an unparallel complexity of the A Plant. Think about a compressor or pump manufacturers. Hundreds of individual parts get assembled and fabricated together to create a finished product. A plants had more of a convergent rather than a divergent flow of the product routing. When one of the parts is missing we can't complete the assembly - we can't sell it despite of the significant investment already made into the inventory. It is either perfect or it isn't useful. A-plants work well within the Pull system but often its size (number of components) requires a well-developed process and need to be supported by the software to make the operation efficient. Unfortunately, most ERP systems are better designed for the Push vs the Pull methodology.

Following the A-plants, there are T plants. They have a very similar characteristic to I Plants but with a significant number of options at the end of the process. Appliance manufacturer with many finishing alternatives can be a good example of this plant arrangement.

Many manufacturing operations display a number of combinations of the above types and it is not uncommon to see a V plant with one or more value streams converting into an A type finishing with an I or T arrangement.

Most auto assembly plants are typical I Plants. Multiple assembly points, all organized in a "straight line" / sequential operations. Components added at each step but a very limited number divergent or convergent points during the process. I Plants work well with the pull system especially when the number of finished SKUs is limited. Automotive supply chain and its JIT support works very well within this framework. 


\section{Demand Driven Replenishment POWERED DATA FEED SOLUTION}

In the Article "Flow Management System for Maximizing Business Revenue and Profitability" (Okrasa, Warchalowski, Zawada, 2020, pp.193-206) the authors described a business management methodology utilizing Demand Driven Replenishment approach to managing supply chain activities. FMS focuses first on defining all inventory requirements, utilizing a Theory of Constraints (TOC) based Demand Driven Replenishment (DDR) sizing algorithm, to set up targets for key Finished Goods, Raw Materials and SubAssembly items. These inventory buffers break supply chain dependence between unreliable supplier deliveries, variable customer demand and the plant, providing significant stability for the manufacturing operation. Once inventory buffers are in place, a pull-based replenishment signal, in combination with other customer demand, creates the basis for generating the plant load. More stable plant load creates larger production butch for key resources, minimizes their set up requirements, increases overall plant throughput and often reduces manpower. In addition, improved ability to more often make to stock vs. to variable customer demand increase finished goods availability, improves customer service levels and leads to increase in sales. Overall DDR results in a significant positive impact on business profitability by often reducing operational expenses and driving sales increase at the same time. In addition, DDR, most of the time, results in lower overall inventory levels and / or increased inventory turns.

DDR creates significant business benefits and is a natural extension of the Pull replenishment strategy. One of the main differences is that an inventory stock buffer, unlike a typical Kanban "basket", consists of "on the way" and "on site" inventory quantity. It works very well with all types of operation including the most challenging $\mathrm{V}$ and $\mathrm{A}$ types. The best performance can be achieved when supported by a DDR enabled software especially when a number of buffers (raw materials and / or finished goods) is large (i.e. CMS RoadRunner by CMS Montera Inc.). Like all other approaches this inventory management process requires a number of behavioral changes in order to be implemented successfully: 
1. No more forecasting at the SKU level. Since this process is ineffective at best and the consumption-based replenishment signal is sufficient to generate "what to buy" and "what to make" suggestions forecasting can be discontinued. An exception is the New Product introduction activities which normally doesn't have enough consumption history to rely on.

2. SKU level inventory management. It is critical to monitor and manage all inventory at the lowest possible BOM level. It is not enough to understand the overall level of inventory (i.e. two months of a product). It is imperative to calculate an Inventory Imbalance identifying all SKUs with the quantity above or below its target (i.e. we have $\$ 2 \mathrm{M}$ too much and $\$ 1 \mathrm{M}$ below the buffer target of the following SKUs).

3. Follow the buffer signal. One of the best characteristics of the DDR software is its ability to automate "what to buy" and "what to make" signals. Trust in the system, however, is imperative to be able to take an advantage of its functionality. Often human behaviour is an obstacle to this very benefit.

4. Utilize Dynamic Feedback Loop. DDR Software recognizes the need to monitor buffer targets and routinely resize them. Seasonality, changing customer demand, poor or improved supplier performance, manufacturing plant effectiveness, all impact buffering needs and need to be administered. Proper supervision of all these parameters often creates a daunting task for the supply chain team.

As already stated in the above-mentioned article, FMS system utilizing a DDR approach, minimizes system dependence by positioning inventory in key supply chain points (i.e. Raw Materials, Finished Goods, customer locations, etc.), provides better protection from on-going disturbances, monitors sources of system variability and allows the entire system function at a higher performance level.

While the business benefits of DDR implementations are obvious there are several obstacles to their implementations ( Seethaler, 2016, pp.4-12):

1. Significant monetary investment for the DDR software module. Not only it is not easy to find a good software that can properly enable DDR methodology, but it is also expensive to cover its licensing and implementation costs. Lack of understanding of this approach by almost 
all ERP vendors decreases natural competition within this arena resulting in higher than average software price points.

2. Availability of in-house IT resources to support the implementation. Most implementations require a certain level of system integration that often cannot be implemented only by the software vendor. The more interactions and data sharing with our ERP system is required the more help the software vendor will need to properly architecture our system needs.

3. IT infrastructure hardware and software expenditures.

In most cases an update of the IT infrastructure is needed to enable proper installation and new system performance. New operating software modules or versions (i.e. Windows server, SQL server, etc.) are necessary to even start the process. Same issues concern existing servers, computers, terminals, and other peripherals.

4. Extensive supply chain and production planning team training.

New methodology and a new IT system will always require building new skills and processes. This knowledge is critical to a successful mplementation and obtaining expected business benefits.

5. Implementation project duration.

Timeframes needed to move the organization from the project kickoff to the independent performance is often measured in multiple months if not years. Unless there is an organizational focus on this particular initiative there is always a danger of project failure due to multiple business priorities, team multitasking, business environment changes, ect. All of these factors may negatively impact project success rate. All of the obstacles above lower the rate of DDR methodology acceptance and minimize business benefits of all organizations where this solution could be successfully implemented - especially within the $\mathrm{V}$ and A plant environments.

CMS Montera developed a methodology to offer DDR based supply chain management service. In order to maximize business benefits, it relies on their, cloud based, CMS RoadRunner Rx software module. Client interactions normally follow these steps:

1. Data Analysis and Modeling.

CMS' team helps to define, generate and verify integrity of historical ERP data extract (transactions and information) in order to create a DDR Simulation. CMS team analyzes data, defines buffering 
requirements and calculates buffer sizes. This modeling exercise demonstrates a difference between "what has happened" and "what could have happened" based on DDR principles. The value proposition in terms of inventory $\$$ savings, increased inventory turns, product availability increase, lead time reduction and a potential revenue increase gets generated.

2. Process design and set-up.

Upon a successful acceptance of the simulation results, process design and software set-up phase is initiated. CMS generates feedback process and finalizes inventory design using DDR principles (SKUs, locations, sizes, etc.). It sets up / updates RoadRunner software and clients inventory scorecard hosting services. In addition, project team is defined, and a short DDR methodology training is delivered.

3. Replenishment and Improvements.

An initial inventory replenishment service including daily communication of "what to buy" and "what to make" suggested quantities is triggered. In addition, an on-going communication mechanism gets defined together with process stakeholders involved.

4. Process Feedback and Optimization.

In this phase the Inventory Scorecard gets set up, KPIs confirmed and adjusted as needed and process monitoring frequency defined. Performance feedback in terms of inventory turns, inventory imbalance, product availability and revenue is evaluated.

5. On-going DDR outsourcing service.

Finally, an on-going outsourcing service driving inventory improvements starts.

This first of a kind outsourcing service mitigates most of the implementation obstacles specified earlier but specifically:

- $\quad$ Significant monetary investment for the DDR software module.

CMS' outsourcing service is priced based on the monthly subscription model fundamentally changing CAPEX requirements to a much lower periodic operating expense. No more licensing and maintenance fees.

- Availability of in-house IT resources to support the implementation. While this issue doesn't entirely disappear the implementation timeframe 
gets significantly reduced. This in turns makes this requirement much easier to manage.

- IT infrastructure hardware and software expenditures.

This normally necessary condition is being removed entirely. Cloud based software offering diminishes this need all together.

- Extensive supply chain and production planning team training.

Under this outsourcing service vast majority of activities are performed by the team of CMS' experts. Client's team needs more of an awareness than an extensive process training significantly reducing this obstacle.

- Implementation project duration.

Project duration gets vastly condensed and its complexity immensely reduced. This increases chances of success for the entire initiative. Here is the full set of services performed as part of the DDR Data Feed service:

- Manage Component / Raw Materials and Finished Goods Buffer Inventories

- Design and re-size levels, locations \& optimize turns

- Help with New Products replenishment introductions

- Communicate Daily Order Requirements-"What to Buy \& Make"

- Monitor Supply Chain Pipeline Status (ensure right supply)

- Design and maintain Inventory Performance Scorecard

- Evaluate Vendors and Purchasing Team's Performance

- Generate slow and non-moving inventory reports

- Create Purchase Order Expedite List

DDR Data Feed project implementations have obviously a significant impact on the organizational performance, its processes and people involved. Each project requires building a relationship, trust and good understanding of everyone's roles and responsibilities. Following change management principles and well established methodology is a must. 


\section{Conslusion}

Modern enterprises must be flexible, innovative and agile to develop. These characteristics of an enterprise are shaped by management through change. Change determines the improvement of the organization and organizations that are open to change are largely successful. One of the areas where there are unused opportunities for organizations is organizational culture. Shaping the organizational culture may support the growth of the organisation. It is about adapting to change in order to maintain and even increase efficiency of the organization. On one hand an organization has an organizational culture and on the other the organization is a culture. That organizational culture is a systemic feature of an organization as a social system. Moreover when an organization becomes an organizational culture, it is a process in which the organization builds up and develops.

The global economy functions at present in a period of dynamic changes resulting from deep structural changes, high frequency of crises, including the crisis caused by the COVID-19 pandemic, as well as technological progress and broadly understood digital transformation. These challenges require measures to adapt to changing conditions, although it is not always successful and it is possible. An example of a pandemic-induced supply chain disruption has had profound implications for producers and consumers around the World. It turned out that enterprises were not flexible enough to manage this new situation. The supply chain of the future will need to be agile, flexible, efficient, resilient and digitally networked for improved visibility. New methods and tools are being rapidly developed and broadly applied. DSNs, Push-pull strategy, VATI Analysis, DDRs mentioned in this paper are only some of them. It is important that apart the application of new tools and technologies, the enterprises will develop new business models following the need of the organization changes in today's business reality. The above-mentioned conditions as well as changes in the supply chain management strongly influence the changes in the organizational culture of enterprises and constitute a challenge for the development and efficient management of change. 


\section{REFERENCES}

Covid-19. (2020). Managing supply chain risk and disruption. Deloitte Canada. Biedenbach T., Soderholm A. (2008). The Challenge of Organizing Change in Hypercompetitive Industries: A Literature Review, Journal of Change Management , Vol. 8, Issue 2, pp. 123-145.

Ernst \& Young. (2020). Global board risk survey, from www.ey.com.

Kalinzi Ch., Outsourcing (Logistics) Services and Supply Chain Efficiency - A Critical Review of Outsourcing Function in Mukwano Group of Companies, Journal of Outsourcing \& Organizational Information Management, Vol. 2016, p. 3.

Komorowski, P. (2020). Role of digitization for German savings banks, in: Digitalisierung - „the next challenge“ für Sparkassen Handlungsfelder zur künftigen Gestaltung des Geschäftsmodells. in E. Utz (ed.), Sparkassen, Deutscher Sparkassen Verlag, Stuttgart 2020, pp. 329-343.

Korhopnen, J., Honkasalo, A., Seppälä, J. (2018). Circular Economy: The concept and its limitations, Ecological Economics Journal 143, pp. 37-46.

Kroes, J. R. (2007). Outsourcing of supply chain processes: Evaluating the impact of congruence between outsourcing drivers and competitive priorities on performance, Georgia Institute of Technology, p. 1.

Minculete, G., Olar, P. (2016). "Push" and "Pull" systems in supply chain management. Correlative approaches in the military field, Journal of Defense Resources Management, Vol. 7 Issue 2 (13), pp.165-172.

Murray, A., Skene, K., Haynes, K. (2017). The Circular Economy: An interdisciplinary exploration of the concept and application in a global context, Journal Business Ethics 140, pp. 369-380.

Okrasa, W., Warchalowski, J., Zawada, P. (2020). Flow management system for maximising business revenue and profitability, Statistics in Transition, New Series 21(5), Polish Statistical Association, pp.193-206.

Sachs, J. (2015). The Age of Sustainable Development, Columbia University Press, New York.

Silva, T. F. G., Goncalves, A. T. P., Leite, M. S. A. (2014). Logistics cost management: insights on tools and operations, International Journal of Logistics Systems and Management, Vol. 19, No. 3. 2014, pp. 329-343.

Seethaler, F. (2016). Assessing the Impact of DDR Programmes: Possibilities and Challenges, UN University, New York, pp.4-12. 